Relations industrielles

Industrial Relations

\title{
Bureau international du travail, Les normes internationales du travail
}

\section{Pierre Verge}

Volume 48, numéro 2, 1993

URI : https://id.erudit.org/iderudit/050864ar

DOI : https://doi.org/10.7202/050864ar

Aller au sommaire du numéro

Éditeur(s)

Département des relations industrielles de l'Université Laval

ISSN

0034-379X (imprimé)

1703-8138 (numérique)

Découvrir la revue

Citer ce compte rendu

Verge, P. (1993). Compte rendu de [Bureau international du travail, Les normes internationales du travail]. Relations industrielles / Industrial Relations, 48(2),

372-374. https://doi.org/10.7202/050864ar

Tous droits réservés @ C Département des relations industrielles de l'Universite Laval, 1993
Ce document est protégé par la loi sur le droit d'auteur. L’utilisation des services d'Érudit (y compris la reproduction) est assujettie à sa politique d'utilisation que vous pouvez consulter en ligne.

https://apropos.erudit.org/fr/usagers/politique-dutilisation/ 
cependant que l'arrêt de la Cour suprême ne peut pas trouver application par simple analogie. Il faudra que le dommage résulte de la rupture du contrat prévue à l'article 124, puisque c'est dans la mesure où le droit a pris naissance dans la convention collective que la Cour a reconnu le pouvoir à l'arbitre de grief.

L'auteur termine ce chapitre par le constat qu'à ce jour moins de la moitié des salariés ayant été l'objet d'un congédiement sans cause juste et suffisante ont été réintégrés. Cependant il propose dans sa conclusion de l'ouvrage un tempérament aux déductions que l'on pourrait tirer de ces informations en soulignant qu'il faut lire ces données en perspective avec le nombre de plaintes déposées auprès de la Commission des normes du travail et en tenant compte de l'effet dissuasif que l'existence d'un tel recours comporte. Bien qu'il ne s'agisse pas de l'objectif premier de ce traité, cette conclusion de l'auteur est intéressante. Quant à l'analyse-synthèse du contentieux entourant le recours de l'article 124 L.N.T., nous croyons que le professeur Laporte a réussi à exposer l'état du droit sur cette question de façon claire et compréhensive, ce qui fait de ce traité un outil de référence utile pour l'ensemble des pratriciens et praticiennes.

Diane L. DEMERS

Montréal

Les normes internationales du travail, Manuel d'éducation ouvrière, troisième édition (révisée), Genève, Bureau international du travail, 1992, 143 p., ISBN 92-2-206445-3

Manuel d'éducation ouvrière, cet ouvrage présente l'activité normative de l'Organisation internationale du travail. Après avoir retracé les origines de l'institution savait-on qu'au milieu du siècle dernier un industriel français du nom de LeGrand avait fait campagne en vue de faire adopter certaines normes du travail européennes? -, il en décrit la structure tripartite et les organes, de même que le mode d'élaboration et d'adoption des conventions et recommandations.

Suit une synthèse de l'objet et du contenu de ces instruments : à la fin de 1990 , on comptait cent soixante et onze conventions et cent soixante-dix-huit recommandations traitant notamment de la liberté syndicale, de la liberté du travail, de l'égalité de chances et de traitement entre la main-d'œuvre masculine et la main-d'œuvre féminine, de l'emploi, de la formation professionnelle et du placement, de l'inspection et des statistiques du travail, de la négociation collective, du tripartisme, du salaire et autres conditions de travail, de la santé et de la sécurité du travail, de la cessation de la relation de travail, de même que d'aspects majeurs de la sécurité sociale - soins médicaux, indemnité de maladie, prestations de maternité, d'invalidité, de vieillesse, de chômage et prestations en cas d'accidents du travail et de maladies professionnelles.

Une seconde moitié, plus ou moins, du texte expose les modes d'application de ces normes : soumission initiale aux autorités gouvernementales compétentes, pour ratification éventuelle, ou, à tout le moins, en vue de la confrontation périodique de l'état de la législation interne et de son contenu : procédure normale de contrôle, de l'accomplissement des obligations des États membres - examen des rapports gouvernementaux 
par la Commission d'experts et étude du rapport de cette dernière par la Conférence internationale du travail, le dialogue entre l'OIT et les États membres étant en outre à l'occasion favorisé notamment par voie de «contrats directs » entre les gouvernements et des représentants du directeur général du BIT. Un chapitre distinct expose les procédures de réclamation et de plainte à l'encontre d'un État membre qui n'a pas assuré l'exécution d'une convention à laquelle il avait adhéré, de même que des voies d'enquête ou d'étude spéciales également ouvertes. Un autre traite spécifiquement de la protection de la liberté syndicale et des droits syndicaux; rôle respectif de la Commission d'investigation et de conciliation en matière de liberté syndicale et du Comité de la liberté syndicale, qui a déjà eu à connaître près de mille six cents cas et dont la jurisprudence touchant à des sujets cruciaux comme le droit de grève, les clauses de sécurité syndicale, l'osmose droits syndicaux-libertés publiques est succinctement résumée.

Une tentative d'apprécier l'influence de ces normes internationales du travail sur la législation interne des divers pays du monde clôt la présentation, à laquelle se greffe une liste thématique des conventions et recommandations par l'OIT à la fin de 1990.

Toute cette matière donne lieu à un exposé simple et essentiel, d'une grande clarté, grâce notamment à des illustrations factuelles appropriées. En plus d'indications bibliographiques essentielles, on trouve à la fin de chaque chapitre des sujets de discussion et des exercices de groupe qui invitent à un prolongement actif et communautaire de la lecture.

Ce contenu et ces caractéristiques permettent de poser que l'ouvrage accomplit sa mission de guide à l'intention des chargés de cours d'éducation des travailleurs. Plus largement, il constitue sans doute la synthèse la plus accessible et utile de l'activité normative de l'OIT et de sa portée dans la perspective de la formation initiale du spécialiste des relations du travail ou du juriste s'orientant vers ce domaine. À ce titre, il mérite l'attention des universitaires canadiens et québécois désireux d'une présentation équilibrée et ouverte des aspects fondamentaux du droit et des institutions du travail. Comme le fait observer la conclusion, grâce notamment à l'efficacité des procédures de promotion et de contrôle de ces normes internationales du travail, ces dernières ont exercé et continuent d'exercer leur influence, directe et indirecte, sur la gestation de la législation des pays industrialisés, comme de ceux en développement.

Plus particulièrement, l'ouvrage permet de s'initier à des techniques d'action dont la souplesse, justifiée par la disparité des situations nationales, $n$ 'est pas dénuée d'intérêt pour qui se préoccupe plus généralement de politique législative. On songe ici, en particulier pour ce qui est de l'élaboration de la norme elle-même, à la distinction de la convention et de la recommandation, à celle entre instrument de portée régionale ou professionnelle et celle de portée universelle, aux « clauses d'application graduelle » d'une convention ou encore, le cas échéant, au caractère promotionnel de celle-ci (voir p. 32-39, en particulier). La gamme d'intervention se prête à un jeu encore plus délicat lorsqu'il s'agit d'assurer l'application de la norme internationale : effets d'entraînement escomptés de sa soumission aux autorités nationales (p. 75 et p. 124), gradation des instances et des moyens d'intervention face à une situation d'inexécution de la norme (p. 86-96 et, s'agissant spécifiquement de la liberté syndicale, p. 110-113). 
Nécessaire initiation à une loi internationale, indissociablement liée aux droits fondamentaux de la personne, « dont l'autorité transcende les frontières » (p. 85).

Pierre VerGe

Université Laval

Alternatives to Lean Production. Work Organization in the Swedish Auto Industry, by Christian Berggren, Ithaca, New York, ILR Press, 1992, 286 p., ISBN 0-87546-193-X

Lorsque Womack, Jones et Roos publiaient, en 1990, The Machine that Changed the World, un avertissement était lancé : l'industrie occidentale court à sa perte si elle ne fait pas siennes les techniques de production contractile (lean production). Pensées, développées et appliquées au Japon, ces techniques étaient décrites comme les seules unissant à la fois les avantages de la production de masse (e.g. réduction des coûts de production) et ceux de la production artisanale (e.g. qualité du produit). L'on pronostiquait alors une véritable révolution qui ferait du modèle de production japonais un patron standard destiné à marquer le XXI ${ }^{c}$ siècle. De ce contexte, la contribution de Berggren tire l'essentiel de sa pertinence. L'auteur conteste en effet les avancées de Womack, Jones et Roos et cherche à contrecarrer un courant qu'il qualifiera de propagandiste.

Puisant dans de riches matériaux empiriques (six monographies d'entreprises de l'industrie automobile suédoise réalisées par l'auteur font l'objet d'une attention particulière et sont complétées par quatorze autres regroupant le Japon, les États-Unis et quelques pays d'Europe), Berggren offre une vision différente et critique du modèle japonais d'une part et, d'autre part, présente une variété de modèles d'organisation technique et sociale du travail qui contrastent avec le premier sans se mouler à la production de masse ou artisanale. En outre, il cherche à évaluer les différentes possibilités de concilier les exigences de la production avec les capacités et besoins de l'homme.

Contrairement aux dires de nombreux analystes dont Krafcik et MacDuffie, Berggren soutient qu'il n'existe pas de rupture entre le taylorisme et le toyotisme; le second constituerait plutôt selon lui une extension complexe du premier. Pour appuyer sa thèse, il explique dans les moindres détails les différences et similitudes caractérisant les deux modes de production comparés et en retient trois éléments importants. Le premier renvoie au caractère hautement standardisé des deux systèmes de production ainsi qu'au maintien solide des prérogatives patronales. Le second a trait à l'importance du rôle du contremaître et à la faible portée des recommandations syndicales dont le contremaître se fait d'ailleurs le héraut. Le troisième élément, traité avec plus de nuances, concerne les relations entre le salarié et la compagnie. Berggren y voit une continuation et un changement en regard du taylorisme dans la mesure où le toyotisme parvient à favoriser l'identification des travailleurs vis-à-vis de la compagnie par le biais de politiques individuelles.

Quant aux solutions de rechange au modèle de production contractile japonais, l'auteur nous propose une variété de modèles, étudiés en Suède, qui se situent aux 